\title{
A mediação de Equipamentos para a Educação Ambiental do Eixo Atlântico na relação Comunidade-Dieta-Alter- ações Climáticas
}

The Mediation of Environmental Education Facilities of the Atlantic Axis in the relation between Community-Diet and Climate Change

Sara Costa Carvalho', Pablo Angél Meira Cartea² e Ulisses Miranda Azeiteiro 1.

1. Universidade de Aveiro (Portugal). 2. Universidade de Santiago de Compostela (Galiza-España)

\begin{abstract}
Resumo
Os padrões de consumo alimentar influenciam significativamente as Alterações Climáticas $(A C)$, as quais têm vindo a "mediterranizar" o norte da Península Ibérica. Sendo a mudança de comportamento a componente mais crítica e a dieta um dos aspectos cujas decisões pessoais mais podem influenciar essa mudança, é importante que os equipamentos para a educação ambiental (EqEA) atuem a nível comunitário. O projeto visa promover os EqEA como mediadores entre AC-padrões alimentares e as comunidades locais, na Euroregião do Eixo Atlântico (Galiza e Norte de Portugal). Através da investigação-ação e de três níveis de EqEA - um EqEA piloto, um conjunto restrito de EqEA, um conjunto não limitado - haverá uma intervenção socio-pedagógica. Os dois primeiros níveis participarão em grupos de I-A e em ações de formação, e todos os níveis receberão formação. Esperase que este projeto contribua para a tomada de consciência da relação entre a dieta e as $A C$, assim como para uma mudança de comportamentos alimentares nos cidadãos e comunidades envolvidas relativamente às $A C$.
\end{abstract}

\section{Astract}

Current patterns of food consume have high influence on Climate Change (CC), which has an ongoing "Mediterranization" pattern in the north of Iberian Peninsula. Since the change of behavior is the most critical component in this matter and being diet patterns one of the aspects in which personal decisions most can influence this change, it is important that resources such as the Environmental Education Facilities (EEF) act at a community level. This project aims to promote the EEF as mediators between CC-food patterns and the local communities, within the Euro Region of Eixo Atlântico (Galicia, Spain and the Northern Portugal). Throughout a methodology of investigation-action (I-A) and using three levels of EEF - a pilot EEF, a restrict set of EEF, and a wider set of EEF - it will be developed a sociopedagogical intervention. The first two levels will participate in groups of $I-A$ and all the levels will have training courses. It is expected that this projects leads to an effective change of behavior among citizens and communities concerning CC. 


\section{Palavras chave}

Equipamentos para a educação ambiental; Alterações Climáticas; Euroregião do Eixo Atlântico; Descarbonização; centros de educação ambiental.

\section{Key-words}

Environmental education facilities; Climate Change; Euroregion of the Atlantic Axis; decarbonization; environmental education centers.

\section{Introdução}

A presente comunicação divulga um projeto de Pós-doutoramento recentemente aprovado pela Fundação para a Ciência e Tecnologia, Portugal, na área da Educação para as Alterações Climáticas (AC). Tal projeto tem como contexto educativo os Equipamentos para a Educação Ambiental (EqEA) e incide nas implicações da nossa dieta no fenómeno das AC.

O desenho do projecto partiu da seguinte hipótese investigativa: Os EqEA não estão a trabalhar o tema das AC de acordo com a urgência do problema, incluindo a causa difusa relacionada com a produção e consumo de alimentos. Tal lacuna repercute-

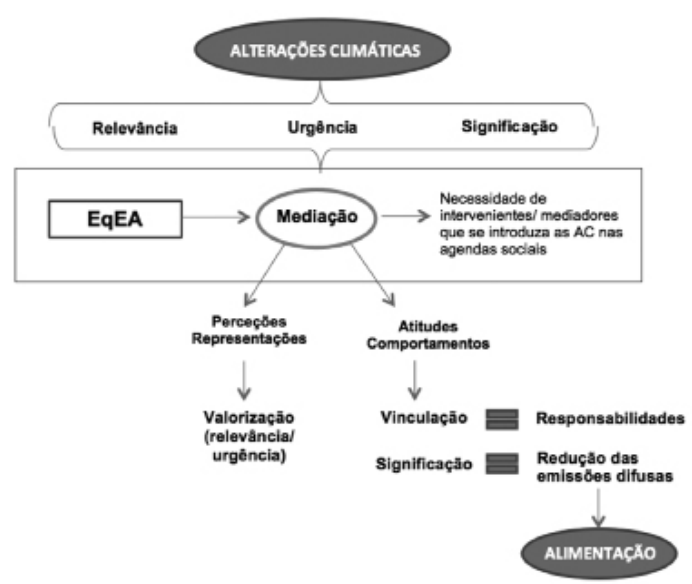

-se nos destinatários das atividades pedagógicas e na população local de cada EqEA, colocando-se a sub-hipótese investigativa de que esses destinatários, em geral, não percepcionam a relação entre AC e o tipo de dieta que praticam diariamente.

Assim, o projeto tem como objectivo geral, ajudar os EqEA a resignificar as atividades e projetos em torno da questão das Alterações Climáticas, no sentido da descarbonização dos estilos de vida, e em especial, através da dieta (estilo de alimentação). Isto é, pretende-se resgatar o conceito dos EqEA como mediadores entre o problema global das $A C$ e as comunidades locais (Figura 1).

O projeto tem como objectivos específicos:

- Constituir uma rede de EqEA na Euroregião do Eixo Atlântico para intercâmbio, formação especializada e inovação pedagógica, em torno à descarbonização nos estilos de vida das comunidades envolventes dos EqEA;

- Estudar os tipos de intervenção pedagógica que estão a ser desenvolvidos actualmente pelos EqEA incluídos na rede, como estratégia de diagnóstico;

Figura 1: Quadro conceptual da investigação 
- Estudar a perceção dos destinatários das atividades pedagógicas (da comunidade local) acerca da relação entre $A C$, produção de alimentos e dieta (consumo);

- Implementar uma intervenção pedagógica com os EqEA da rede formada, que seja adequadamente contextualizada ao entorno de cada EqEA e que permita intervir sobre os estilos de vida da população;

- Avaliar o plano de intervenção pedagógica, incluindo os impactos educativos e sociais das ações desenvolvidas, através de instrumentos adequados que permitam uma avaliação de seguimento e final;

- Atingir uma mudança de comportamentos nos cidadãos e nas comunidades envolvidas.

\section{Estado da Arte}

Os padrões de consumo relacionados com a alimentação afetam as emissões de gases de efeito de estufa (GEE) (FABER et al., 2012). Em concreto, estima-se que os alimentos sejam responsáveis por cerca de $30 \%$ das emissões globais (GarnetT, 2011).

Tais padrões de consumo alimentar ou dietas de baixo Vs. elevado carbono incluem opções como a preferência ou não por produtos locais e sazonais, por produ- tos com certificação biológica, dieta com reduzida ou elevada quantidade de carne (Boer, Witt \& Aiking (2016) e atenção ao desperdício de alimentos (BLANCo et al., 2014).

No contexto europeu e, em particular na Península Ibérica, as Alterações Climáticas (AC) tendem a originar uma "mediterranização" do norte (UA, s/d). As emissões também ameaçam as tradições e culturas locais (Adger et al., 2012) como, por exemplo, a prática pecuária com raças autóctones, no interior da Galiza (UA, s/d) e do Norte de Portugal (NP).

Não obstante, cresce o ceticismo sobre as $A C$ em alguns países desenvolvidos (CAPSTICK et al., 2015), para além de haver lacunas em conhecimentos e ações (MElRA-CARTEa et al., 2011). O estudo de Boer, WITT \& AIKING (2016), conclui que apenas $12 \%$ dos holandeses e $6 \%$ dos americanos reconhece que a redução substancial de carne é das medidas mais efectivas para a mitigação das AC. No entanto, o consumo de carne de acordo com as recomendações dietéticas saudáveis consegue reduzir as emissões provenientes da produção destes produtos em aproximadamente de 15 a $25 \%$ do total de GEE, segundo o exemplo sueco (Hallstrom, Roos \& BORJESSON, 2014). No NP, estudantes universitários representam as AC baseando-se na cultura comum e não na formação científica (NEVES, 2011) e estudantes em Espanha e México raramente relacionam 
este problema com a alimentação (ARTO-BLAnco \& MeIra-Cartea, 2017).

Perante este panorama, a Associação Transfronteiriça da Euroregião Eixo Atlântico do Noroeste Peninsular e, em particular, a Agência de Ecologia Urbana tem apoiado projetos de mitigação e adaptação às AC como o CLIMATLANTIC (SoRROSAL, 2010) e iniciativas no Programa INTERREG V-A Espanha-Portugal 20142020 (BAleiras, 2009). Em Portugal, para além da Estratégia Nacional de Adaptação às $A C$, foi implementado o Projeto ClimAdaPT.Local (Estratégias Municipais de Adaptação às AC) (APA, s/d), Na Galiza, também existem planos municipais e sectoriais como na área agro-florestal.

Nestes processos, a mudança de comportamento dos indivíduos e organizações é a componente mais crítica (VAUGHTER, 2016). Então: "A tarefa de um programa educativo para mitigar as AC e promover a adaptação às suas consequências inevitáveis, é representar um problema colectivo global à escala individual e social" (IPCC, 2014: 69). Através de uma abordagem transversal é possível tornar visíveis as alternativas ecológicas e implicações técnicas, económicas, éticas, culturais e políticas (ARTO-Blanco \& MeIra-Cartea, 2017).

Por exemplo, no que concerne à transição para uma dieta de baixo carbono, baseada nomeadamente na redução de consumo de carne, a pesquisa de LAESTADIUS et al. (2014) mostra que é necessária uma colaboração nomeadamente entre organizações não governamentais (ONG) de diferentes âmbitos e não apenas de ONG de ambiente para fortalecer a divulgação e o envolvimento cidadão nesse sentido. Assim, as intervenções deverão ser baseadas na participação, a relevância e a interconectividade (Allen \& Crowley, 2017) através de sessões práticas (ColuINS \& Ison, 2009, McCRUm et al., 2009) e envolvendo também adultos (ex.: grupos de profissionais) e seniores (VAUGHTER, 2016).

Estruturas de educação não formal como os equipamentos para a educação ambiental (EqEA) podem favorecer uma educação ambiental sociocrítica por serem, nomeadamente, referências estáveis para a comunidade local (SERANTES, 2011 e SERANTES \& BARRACOSA, 2008). Os EqEA estão assim incluídos na designação de recursos ou instrumentos de corpo físico, intelectual ou metodológico que facilitam a consecução dos objectivos da EA (SERANTES, 2011). No presente estudo, a denominação de equipamentos para a educação ambiental (EqEA) é tomada, formalmente, como se tratando de um conjunto de iniciativas heterogéneas constituídas por vários elementos (Figura 2).

De acordo com a figura 2, os EqEA têm: 1) instalações com objectivos próprios da EA; 2) projecto educativo com actividades definidas; 3) equipa educativa, estável e profissionalizada; 4) recursos materiais, 


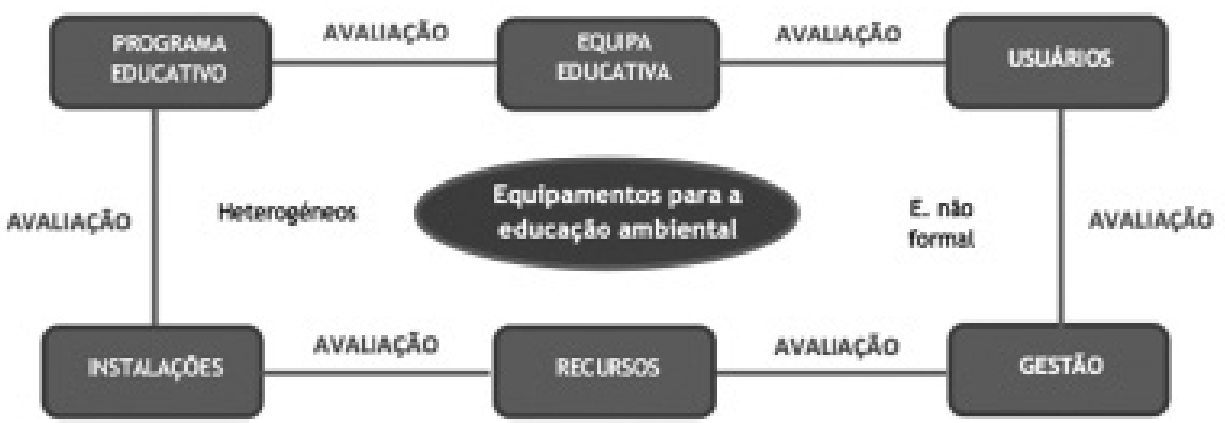

Figura 2. Definição de equipamentos para a educação ambiental (Serantes, 2011)

humanos e metodológicos; 5) modelo de gestão sustentável ambientalmente; 6) com sistema avaliação do projecto; 7) sendo este adaptado a cada tipo de usuários. Perspectivando a dimensão de mobilização local, os equipamentos são encarados como potenciais dinamizadores sociais (Serantes \& Barracosa, 2008), no sentido do desenvolvimento comunitário e o reforço de coesão social. Segundo esta óptica de mobilização social e da abordagem sociocultural nas atividades, os equipamentos poderão ter maior ou menor "peso" na sociedade local ou seja, um nível maior ou menor de impacto social. Este conceito, por sua vez, tem associados três critérios-chave: nível de participação dos usuários; contacto com a realidade local; duração das acções (Serantes \& BarRacosa, 2008).

$\mathrm{Na}$ abordagem sociocrítica ao fenómenos das $\mathrm{AC}$, EqEA como os museus também estão posicionados para funcionarem como agentes de governância para as $A C$ pois o público confia na informação livre de influência política (Allen \& Crowley,
2014) e podem promover a participação em ações sobre AC (CAMERON, Hodge \& SALAZAR, 2013).

No Eixo Atlântico são escassos os trabaIhos em EA e em EqEA, e além disso tão pouco existem instrumentos estratégicos específicos para este sector (MEIRA \& PINTO, 2008). Sabe-se, no entanto, que as AC são abordadas de forma residual em EqEA (CArvalho et al., 2011; Carvalho, 2015; SeRANTES, 2011). A investigação nesta região também vem da sequência da pesquisa de doutoramento realizada por Carvalho (2015), sobre a integração de aspetos biofísicos e socioculturais locais em EqEA do Eixo Atlântico.

Enquadrando o surgimento desta Euro-região, já desde períodos ancestrais que os povos do espaço geográfico da atual Galiza e do Norte de Portugal tiveram experiências comuns de sobrevivência. Após um largo período de afastamento e com a adesão dos dois Estados à Comunidade Europeia, em 1986, a cooperação voltou 
a ganhar força, incluindo a constituição da entidade que viria a constituir o Eixo Atlântico. A Associação da Euroregião do Eixo Atlântico do Noroeste Peninsular visa assim, o desenvolvimento social, económico, cultural, tecnológico, educativo e científico das cidades e regiões envolvidas.

A participação das cidades da Galiza e do Norte de Portugal foi crescendo, integrando uma estrutura urbana de cidades de maiores dimensões (ex. Porto e Vigo) e de médias dimensões. Em termos de território global, o Eixo Atlântico é dividido por 4 áreas básicas transfronteiriças e 7 áreas adjacentes, ocupando uma superfície de 50700 km2 (Figura 3).

Considerando as semelhanças geográficas e culturais entre Galiza e NP, MEIRA \& PINTO (2008) recomendam o desenvolvimento de iniciativas compartilhadas que

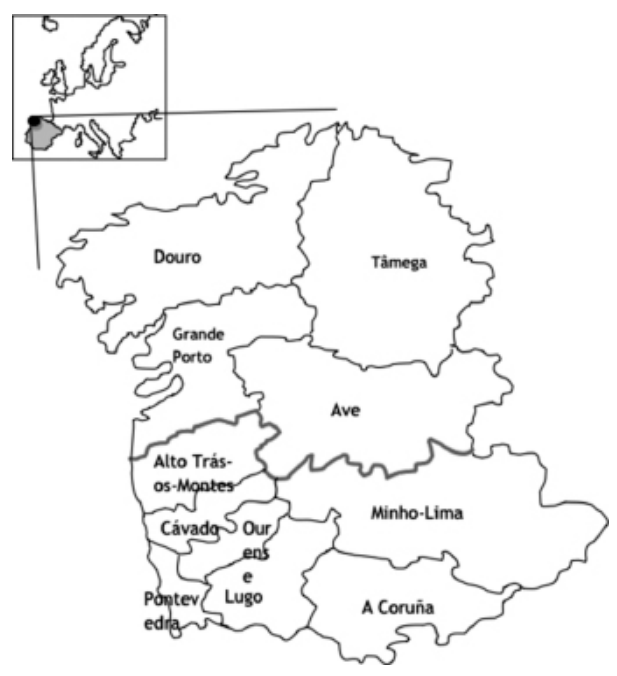

Figura 3. Enquadramento geográfico do Eixo Atlântico na Europa difundam boas práticas de EA e, neste caso, sobre AC.

\section{Metodologia prevista}

Para alcançar o objectivo principal pretende-se realizar uma intervenção socio-pedagógica através de uma rede de EqEA do Eixo Atlântico. O processo a desenvolver seguirá uma linha de investigação-ação (IA) pois pretende-se produzir mudanças na realidade estudada (BISQUERRA, 2000).

Através da I-A do tipo participativo ou cooperativo (SAmpieRI, Collado \& Lucio, 2007) será possível, não só envolver a transformação e melhoria da realidade educativa e social relacionada com as Alterações Climáticas (AC), como também partir de um problema contextualizado e ainda implicar a colaboração dos participantes na detecção de necessidades, no desenho e na implementação das ações.

O projeto contará com três níveis de envolvimento de EqEA da Euroregião do Eixo Atlântico, no papel de mediadores (Figura 4).

De acordo com a Figura 4, o estudo abrangerá:

I. Um EqEA piloto - "caso-zero" - partindo das necessidades socioambientais locais e potencialidades dos recursos naturais endógenos face às $A C$; 


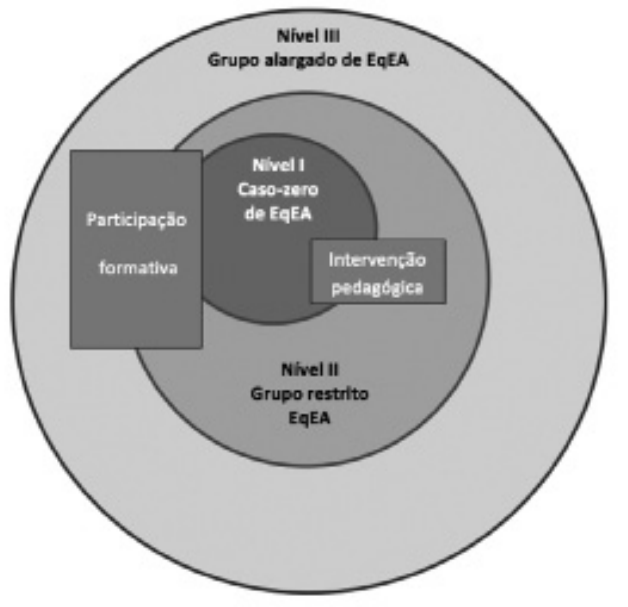

Figura 4. Anéis de níveis de envolvimento de EqEA no projeto

II. Um grupo restrito de EqEA com elevado/médio impacto social, segundo Serantes (2011) e Carvalho (2015); EqEA situados em municípios com estratégias face às $A C$; uns próximos da raia (faixa transfronteiriça) e outros que já abordam o tema das AC;

III. Um grupo alargado de EqEA do Eixo Atlântico, que receberá formação prática ao longo dos vários ciclos do processo de I-A.

No nível I irá participar um EqEA da zona nordeste de Portugal (município de Bragança) que está a dar os seus primeiros passos. O grupo restrito de EqEA do nível II será composto por três equipamentos do Norte de Portugal e quatro centros da Galiza, abrangendo iniciativas da zona costeira e da zona interior do Eixo Atlântico.
Além disso, participarão especialistas em EA e as entidades: Associação Portuguesa de Educação Ambiental (ASPEA), Centro Regional de Excelência de Educação para o Desenvolvimento Sustentável da Área Metropolitana do Porto (CRE Porto) e a Rede de Equipamentos para a Educação Ambiental da Galiza.

Seguindo também o processo próprio da $\mathrm{I}-\mathrm{A}$, interessa que sejam desenvolvidos os quatro principais ciclos sucessivos de planificação, acção, observação e reflexão em espiral autoreflexiva ou dialética (BISQUERRA, 2000), pretendendo a reflexão dar feed-back à "espiral" de I-A.

Para recolher dados ao longo do processo de I-A irá recorrer-se a várias técnicas do tipo qualitativo seguindo um modelo indutivo (BISquerRA, 2000). Tais técnicas incluem:

- grupos de I-A, numa técnica híbrida entre grupos de discussão (Delgado \& GuTIÉRREZ, 1998) e comunidades de práticas (Aguilar \& KRASNy, 2011) e contando cada grupo com elementos da equipa de um EqEA, com a investigadora e, sempre que possível, o líder de um grupo de visitantes;

- análise de documentos institucionais dos EqEA ou realizados pelos participantes nas actividades (ex. textos, desenhos, fotografias, filmes, outros materiais pedagógicos sobre as $\mathrm{AC}$ );

- observação de atividades pedagógicas desenvolvidas no âmbito da intervenção, 
com recurso a gravação de áudio e vídeo e a notas de campo (Bogdan e Biklen, 1994).

O programa de trabalhos está dividido em 7 tarefas:

- Tarefa 1: Aprofundamento da revisão da literatura sobre EA em AC e sobre implicações da dieta/alimentação nas AC e sua relação com a situação sociogeográfica do Eixo Atlântico. Serão consultados documentos, incluindo materiais pedagógicos de outros contextos geográficos, assim como artigos sobre formação de educadores em $\mathrm{AC}$ e trabalho em rede de EqEA;

- Tarefa 2: Constituição da rede de EqEA do Eixo Atlântico em torno da descarbonização nos estilos de vida das comunidades. Como primeiro ciclo da I-A, será planificada a intervenção, começando pela organização de sessões iniciais (oficinas) com os EqEA dos três níveis de envolvimento, numa lógica de trabalho de cenários (previsão de 3 e 6 anos). Esta tarefa inclui ainda a criação dos grupos de I-A e de linhas de orientação envolvendo os EqEA dos níveis I e II;

- Tarefa 3: Pesquisa sobre os tipos de intervenção pedagógica desenvolvidos pelos EqEA do nível II e sobre os problemas socioambientais locais (EqEA dos níveis I e II) relacionados com a relação $A C$ - alimentação. Os grupos de I-A e a análise de documentos (materiais dos próprios EqEA) serão as técnicas principais de recolha de dados. Trata-se de uma estratégia de diagnóstico/avaliação para auxiliar igualmente na planificação da intervenção participada. Os resultados do diagnóstico serão apresentados aos participantes durante os grupos de I-A, para juntar dados e validar informação;

- Tarefa 4: Implementação da intervenção pedagógica com os EqEA da rede formada. A intervenção que inclui atividades e materiais pedagógicos (para destinatários à escolha dos EqEA e de forma regular) será contextualizada em cada EqEA. Aqui também se fará uso dos grupos de I-A. O nível I de EqEA será acompanhado de forma individual no decurso das actividades. Paralelamente serão realizadas ações de formação práticas (oficinas) com os EqEA dos três níveis da rede, com a colaboração de especialistas (em $E A$, em equipamentos e noutras áreas necessárias) e envolvendo as comunidades locais. O ciclo de I-A relacionado com observação (da implementação das atividades e a formação de educadores) irá recorrer às técnicas de notas de campo e análise de documentos;

- Tarefa 5: Reflexão participada com os EqEA sobre as intervenções pedagógicas implementadas, com vista à retroalimentação do processo de I-A. Nesta tarefa são avaliadas as estratégias de intervenção pedagógica (impactos educativos e sociais) e serão efectuados ajustes, através dos grupos de I-A (com os níveis I e II de EqEA) e através de oficinas (com os três níveis); 
- Tarefa 6: Avaliação periódica para balanço da intervenção e do funcionamento da rede temática através de dois momentos de reflexão: a meio do projeto (3 anos) e outro no final (6 anos). Serão realizadas sessões com os três níveis de EqEA (pelos menos duas oficinas, uma com cada região do Eixo Atlântico) para debate de propostas futuras;

- Tarefa 7: Divulgação e publicação dos resultados - organização de seminário internacional (último semestre do projeto) e publicação dos resultados na forma de livro, assim como de três artigos em revistas internacionais com fator de impacto.

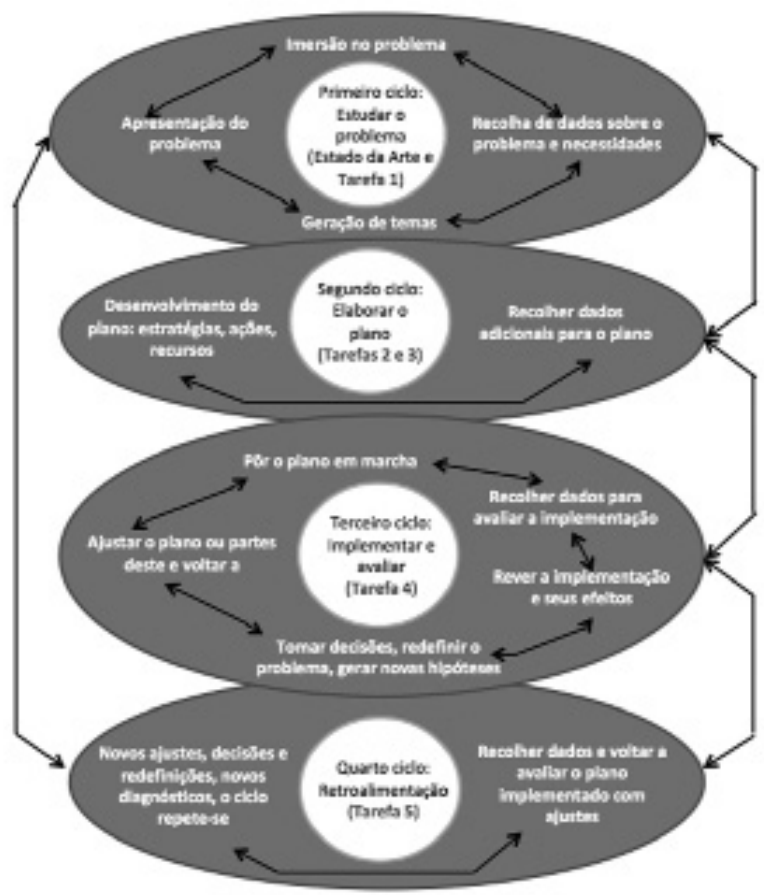

Na Figura 5 é ilustrada a dinâmica das tarefas de 1 a 5 .

De acordo com a figura 5 pode-se observar que o processo de investigação-ação consistirá numa espécie de espiral de conhecimento. Assim, o primeiro ciclo consistirá no estudo do problema; o segundo ciclo, na elaboração do plano; o terceiro ciclo, na implementação e avaliação do plano; e o quarto ciclo, na retroalimentação do processo. Em suma, o projecto Agradecimentos contará com vários ciclos de reflexão participada com os EqEA sobre as intervenções pedagógicas implementadas, com vista à retroalimentação do processo de I-A e à divulgação dos resultados alcan-

Figura 5. Principais fases da investigação-ação e tarefas a desenvolver com os EqEA (adaptado de Sampieri, Collado \& Lucio, 2007)

Espera-se, desta forma, contribuir para uma mudança gradual e efetiva de comportamentos nos cidadãos e comunidades envolvidas relativamente às $\mathrm{AC}$, e em concreto na descarbonização das diversas componentes da dieta. çados. 


\section{Referências bibliográficas}

ADGER, W.N., BARNETT, J., BROWN, K. MARSHALL \& O'BRIEN, K. (2012): “Cultural dimension of Climate Change impacts and adaptation", em Nature Climate Change, 3, pp.112-117.

AGÊNCIA PORTUGUESA DO AMBIENTE (s/d): PR COP21, Adaptação no Acordo de Paris, disponível em http://climadapt-local.pt/wp-content/uploads/2016/02/20151203-PR-COP21_Adaptacao-Acordo-de-Paris.pdf, consultado em 17-06-2016.

AGUILAR, O.M. \& KRASNY, M.E. (2011): “Using the communities of practice framework to examine an after-school environmental education program for Hispanic youth", em Environmental Education Research, 17(2), pp.217-233.

ALLEN, L.B. \& CROWLEY, K. (2014): "Challenging beliefs, practices and content: How museum educators change", em Science Education, 98(1), pp. 84-105.

ALLEN, L.B. \& CROWLEY, K. (2017): "Moving beyond scientific knowledge: leveraging participation, relevance and interconnectedness for climate education", em International Journal of Global Warming, 12(3-4), pp. 299-312.

ARTO, M. \& MEIRA, P.Á. (2017): "Climate literacy among university students in Mexico and Spain: influence of scientific and popular culture in the representation of causes of Climate Change", em International Journal of Global Warming, 12(3-4), pp. 448-467.

BALEIRAS, R.N. (2009): "Caminhos para a Política de Coesão 2014/2020”, em Eixo Atlântico - Revista da Euroregião Galiza-Norte de Portugal, 16, pp. 17-48.

BLANCO, G., GERLAGH, R., SUH, S., BARRETT, J., CONINCK, H.C., MOREJON, C.F., MATHUR, R., NAKICENOVIC, N., AHENKORA, A.O., PAN, J., PATHAK, H., RICE, J., RICHELS, R., SMITH, S.J., STERN, D.I., TOTH, F.L., \& ZHOU P. (2014): "Drivers, Trends and Mitigation". In O. Edenhofer, R. Pichs-Madruga, Y. Sokona, E. Farahani, S. Kadner, K. Seyboth, A. Adler, I. Baum, S. Brunner, P. Eickemeier, B. Kriemann, J. Savolainen, S. Schlömer, C. von Stechow, T. Zwickel and J.C. Minx (eds.). Climate Change 2014: Mitigation of Climate Change. Contribution of Working Group III to the Fifth Assessment Report of the Intergovernmental Panel on Climate Change (New York: Cambridge University Press).

BISQUERRA, R. (2000): Métodos de investigación educativa. Barcelona, CEAC Educación.
BOER, J., WITT A. \& AIKING (2016): "Help the Climate, change your diet: A cross-sectorial study on how to involve consumers in a transition to a low-carbon society", em Appetite, 98, pp. 19-27.

BOGDAN, R. E BIKLEN, S. (1994): Investigação qualitativa em educação: Uma introdução à teoria e aos métodos. Porto, Porto Editora.

CAMERON, F., HODGE, B. \& SALAZAR, J.F. (2013): "Representing Climate Change in museum space and places", em WIREs Climate Change, 4, pp. 9-21.

CAPSTICK, S., WHITMARCH, L., POORTINGA, W., PIDGEON, N. \& UPHAM, P. (2015): “International trends in public perceptions of climate change over the past quarter century", em WIREs Climate Change, 6, pp. 35-61.

CARVALHO, S.C. (2015): Potencialidades e práticas de integração das dimensões sociocultural e biofísica em equipamentos para a educação ambiental: estudos de caso do Eixo Atlântico (Norte de Portugal e Galiza), tese de doutoramento. Santiago de Compostela, Universidade de Santiago de Compostela.

CARVALHO, S.C., AZEITEIRO, U.M. \& MEIRA, P.A. (2011): "Equipamentos para a Educação Ambiental na zona costeira da Euroregião do Eixo Atlântico - Das práticas conservacionistas às sociocríticas", em Journal of Integrated Coastal Zone Management, 11(4), pp. 433-450.

COLLINS, K. \& ISON, R. (2009): “Jumping off Arnstein's ladder: Social learning as a new policy paradigma for Climate Change Adaptation", em Environmental Policy and Governance, 19, pp. 358-373.

DELGADO, J.M. \& GUTIÉRREZ, J. (1998): Métodos e técnicas cualitativas de investigación en Ciencias Sociales. Madrid, Editorial Síntesis.

FABER J., A. SCHROTEN, M. BLES, M. SEVENSTER, and A. MARKOWSKA (2012): Behavioural Climate Change Mitigation Options and Their Appropriate Inclusion in Quantitative Longer Term Policy Scenarios, Dinamarca: Agência Europeia do Ambiente, disponível em http:// ec.europa.eu/clima/policies/roadmap/docs/ main_report_en.pdf, consultado em 17-06-2016.

GARNETT, T. (2011): "Where are the best opportunities for reducing greenhouse gas emissions in the food system (including the food chain)? Em Food Policy, 36, pp. S23-S32.

HALLSTROM, E., ROOS, E. \& BORJESSON, P. (2014): "Sustainable meat consumption: A quantitative analysis of nutricional intake: greenhouse gas emissions and land use from a Swedish perspective", em Food Policy, 47, pp. 81-90. 
INTERGOVERNAMENTAL PLANNEL FOR CLIMATE CHANGE (2014): Climate Change 2014: Mitigation of Climate Change. Contribution of Working Group III to the Fifth Assessment Report of the Intergovernmental Panel on Climate Change [Edenhofer, O., R. Pichs-Madruga, Y. Sokona, E. Farahani, S. Kadner, K. Seyboth, A. Adler, I. Baum, S. Brunner, P. Eickemeier, B. Kriemann, J. Savolainen, S. Schlömer, C. von Stechow, T. Zwickel \& J.C. Minx (eds.)] (New York: Cambridge University Press).

LAESTADIUS, L. I., NEFF, R.A., BARRY, C.L. \& FRATTAROLI, S. (2014): “We don't tell people what to do: An examination of the factors influencing NGO decisions to campaign for reducing meat consumption in light of Climate Change", em Global Environmental Change, 29, pp. 32-40.

MCCRUM, G., BLACKSTOCK, K., MATTHEWS, K., RIVINGTON, M., MILLER, D. \& BUCHAN, K. (2009): "Adapting to Climate Change in Land management: the role of deliberative workshops in enhancing social learning", em Environmental Policy and Governance, 19, pp. 413-426.

MEIRA, P.Á. \& PINTO, J.R. (2008): “A educação ambiental em Galicia e Norte de Portugal: Uma valoração estratégica desde a perspectiva local no Eixo Atlântico", In: L. Cunha e M. Santiago (orgs.), Estratexias de Educación Ambiental: Modelos, experiencias e indicadores para a sostenibilidade local, pp. 31-82. Vigo, Eixo Atlântico do Noroeste Peninsular.

MEIRA, P.Á., ARTO, M. HERAS, F. e SOUTO, P.M. (2011): La sociedad ante el cambio climático. Conocimientos, valoraciones y comportamientos en la población española. Madrid, Instituto de Prevención, Salud y Medio Ambiente, Fundación MAPFRE.
NEVES, A.P. (2011): Representações sociais das alterações climáticas: um estudo sobre a relação entre formação científica e cultura comum em Portugal, tese de doutoramento. Santiago de Compostela, Universidade de Santiago de compostela.

SAMPIERI, R.H., COLLADO, C.F. \& P.B. (2007): Metodología de la investigación (Mexico: Mc Graw Hill).

SERANTES, A. (2011): Os equipamentos para a educación ambiental en Galicia: Análise da realidade e propostas de mellora de calidade, tese de doutoramento, Coruña, Universidade da Coruña.

SERANTES, A. \& BARRACOSA, H. (2008): Contributos dos equipamentos de educação ambiental para as estratégias de acção local. Estudos de caso na Galiza e no Norte de Portugal, In: L.I. Cunha e M.P. Santiago, Estratexias de Educación Ambiental: Modelos, experiencias e indicadores para a sostenibilidade local, pp. 179-200. Vigo, Eixo Atlântico do Noroeste Peninsular.

SORROSAL, A. (2010): "Las redes de cooperación, seña de identidad del Eixo Atlántico”, em Eixo Atlântico - Revista da Euroregião Galiza-Norte de Portugal, 17, pp. 113-120.

UNIÓNS AGRARIAS (s/d): Estrategias para la mitigación del Cambio Climático en el sector agroforestal (Galicia) disponível em http:// www.unionsagrarias.org/lifecambiarocambio/ docs/Estrategias_para_la\%20_mitigacion_del_ cambio_climatico_en_el_sector_agroforestal. pdf, consultado em 17-06-2016.

VAUGHTER, P. (2016): "Climate Change Education: From Critical Thinking to Critical Action", em Policy Brief, 4, pp. 1-4. 